\title{
The Kinematics of Jumping of Globular Springtail
}

\author{
Seiichi Sudo $^{\mathrm{a}^{*}}$, Masahiro Shiono ${ }^{\mathrm{a}}$, Toshiya Kainuma ${ }^{\mathrm{a}}$, Atsushi Shirai ${ }^{\mathrm{b}}$, and Toshiyuki Hayase ${ }^{\mathrm{b}}$ \\ ${ }^{a}$ Faculty of Systems Science and Technology, Akita Prefectural University, Japan \\ ${ }^{\mathrm{b}}$ Institute of Fluid Science, Tohoku University, Japan
}

\begin{abstract}
This paper describes jumping behaviour of the globular springtails. Direct observations on the jumping and climbing behaviour of springtails were conducted in the laboratory. The kinematics of jumping in tiny springtails was analyzed with high-speed video camera system. The vertical climbing behaviour was analyzed in contrast to the jumping behaviour. The jumping performance of springtails was revealed.
\end{abstract}

Index Terms - Springtail Jump, Leaping Organ, Furcula, Aerodynamic Drag

\section{INTRODUCTION}

Most animals are capable of movement, and they move about using legs, wings, or fins. The dynamics of these locomotion of animals are a fascinating subject that has attracted the attention of biologists, engineers, mathematicians, and other scientific workers for a period of many years.

Arthropoda is the largest animal phylum, and is one of tremendous diversity. The name Arthropoda means "joint-footed", and this feature of the arthropods, along with the segmented body structure and the tough outer skin (cuticular exoskeleton), is a distinctive characteristics of the phylum. They are found almost everywhere and make up more than three-quarters of all the living organisms on this planet.

In locomotion such as swimming, walking, running, and jumping, organisms with high Reynolds number tend to be larger and faster moving organisms. The Reynolds number represents the ratio of inertial force to viscous force in the flow. For small Reynolds number, the flow will always be laminar, and viscosity is the resistance of a fluid to flow under the influence of an applied external force.

Springtails are minute insects without wings. Especially, globular springtails have potato-shaped bodies, usually less than about $1 \mathrm{~mm}$ long, and 6 short legs. They are able to perform remarkable jumps into the air. For such springtail jump, viscosity tries to stop it. In general, locomotion is one of the major energetic costs faced by animals and various strategies have evolved to reduce its cost [1]. Therefore, extensive investigations on the jumping performance of insects have been conducted. For example, studies of the energetics and a model of the mechanism in the jump of the rabbit flea [2] and the locust [3] were reported. The effect of muscle properties, leg design and jumping technique for humans, other vertebrates and insects were investigated [4]. The structure of the hind limbs and the kinematics of their movements that propel jumping in plant hopper insects were analyzed [5]. The jumping behaviour in springtails was investigated by high-speed photography methods [6,7].

In spite of the number of investigations, however, there still remains a wide, unexplored domain. Research data on morphological characteristics of leaping organ of globular springtails and the details of jump mechanisms are scarce, and there are many points which must be clarified.

In this study, the jumping and climbing characteristics of a globular springtail were examined using high speed video camera system.

\section{EXPERIMENTAL METHOD}

A schematic diagram of the experimental apparatus to study jumping behaviour of globular springtail is shown in Fig.1. The experimental apparatus consists of the jumping horizontal plate, the optical measurement system, and the analysis system.

The jumping plate made of the wood with dimension $60 \mathrm{~mm} \times 290 \mathrm{~mm}$ wide and $10 \mathrm{~mm}$ in thickness. In order to guarantee the two-dimensional plane in the high-speed images, experiments on springtail jump were carried out in the region of $10 \mathrm{~mm}$ width space. The optical measurement system is composed of a highspeed video camera (Photron FASTCAM-ultima-SE), a control unit, a video cassette recorder (SVO260), a video monitor (PVM-1450), and a personal computer (Endeavor Pro 2000). Micro Nikkor lens (55mm f/2.8S) with the close-up ring was used in the photographing of springtail movements. First, the scales were recorded in the view field of the camera. The view field for recording the whole of the jumping behavior was set in the region of $155 \mathrm{~mm} \times 155 \mathrm{~mm}$. Observations of the early stages of springtail jump was conducted in the region of $6 \mathrm{~mm} \times 3.5 \mathrm{~mm}$. In addition, the view field was modified appropriately. 


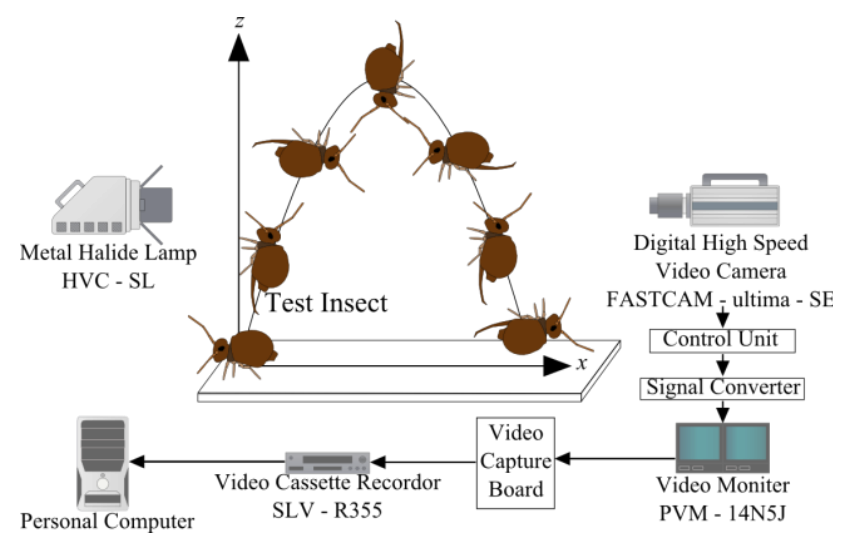

Fig. 1. Experimental apparatus for free jumping analysis of a globular springtail.

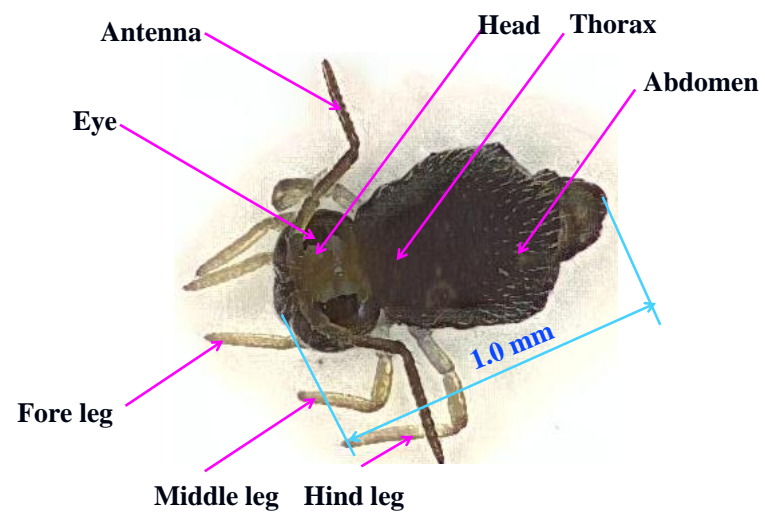

Fig. 2. Photograph of a globular springtail used in the jumping experiment.

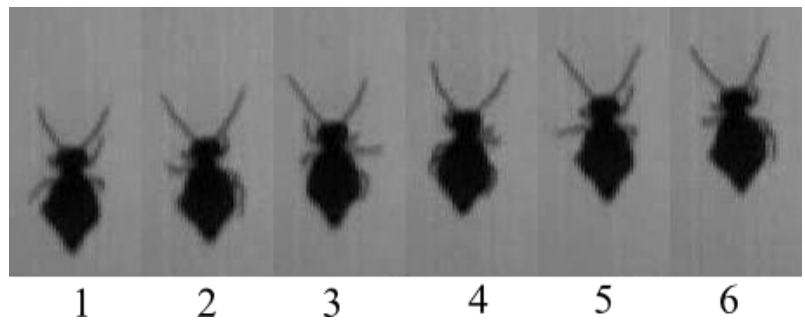

Fig. 3. Video sequence of the springtail climbing.

The globular springtail was released at the position on the plate. Free jumping of the springtail on the plate was recorded with high-speed video camera system, capable of capturing up to 40,500frames per second (fps); typical rates in this study 4,500-27,000 fps. A series of frames of free jumping behaviour of springtail were analyzed by the personal computer. The multiple photograph method was used to determine the jumping trajectory.

The multiple photograph method is a multiple exposure technique on a single picture. The jumping trajectory of the springtail with $1 \mathrm{~mm}$ body length was described as a large number of points. Those points give the position of the insect with sufficient accuracy in the resolution.

Test springtails were collected in the field in Yurihonjo, Japan. They were very small (typically 1.0 $\mathrm{mm}$ in length), and they have a rounded body shape.
Figure 2 shows a photograph of the globular springtail used in this experiment. The head bears a pair of antennae, a pair of eyes, and the mouthpart. The thorax consists of three segments. The abdominal segments fuse making the intersegmental boundaries difficult to resolved as shown in Fig. 2 .

\section{SPRINGTAILS}

\section{A. Collembora}

Springtails belong to a group of insects known as Collembora [8]. Springtails are primitive, minute, wingless insects. They are widely distributed, and are found throughout the year inhabiting moist soil situations. Approximately 2,000 different species of springtails have been identified worldwide. They vary in body shape from elongate to globular. They range in color from white, gray, or yellow to red, orange, purple, brown, or mottled hues. Their abdomens have only 4-6 segments, less than any other insect. Another unusual feature is a tube protruding from the abdomen. The name springtail comes from a forked structure attached to the underside of the abdomen. When this mechanism is released, the abdominal extension snaps back, tossing the springtail into the air. In this study, the globular springtail as shown in Fig. 2 was examined.

\section{B. Vertical Climbing}

Many species of springtails have well developed jumping and climbing behaviour [7]. The effects of variation in body form on the mechanics of terrestrial locomotion were examined using the miniature force platform [9]. Humans, bushbabies, frogs, locusts, fleas jump by rapidly extending a pair of legs [4]. In springtail jump, however, a spring-like appendage (furcula) is used. This unusual locomotor organ is independent of three pairs of legs. In this paragraph, the climbing behaviour of springtail was examined.

Figure 3 displays a sequence of pictures for the climbing behaviour of globular springtail. The pictures show springtail locomotion formed by the legs. The time between successive pictures is $\delta_{t}=50 \mathrm{~ms}$. Figure 4 shows the gait pattern of the globular springtail in climbing of a vertical board. In Fig.4, black bars show the swing phase in which a leg lifts and moves forward. Six characteristic points on the springtail legs were defined by the signs shown in Fig.4. The insects can generate a suitable gait pattern which depends not only on the walking speed, but also on the external load. In general, the insects usually have two kinds of gait patterns, that is, the tripod gait and the metachronal gait [10]. In this experiment, the springtail shows the complicated gait pattern that the tripod and metachronal gaits were mixed. This fact suggests that the climbing of the vertical board accompanies the heavy load. Springtails, however, move the long distance by the jump in short time. In Fig.4, $x_{c}$ is the horizontal distance 


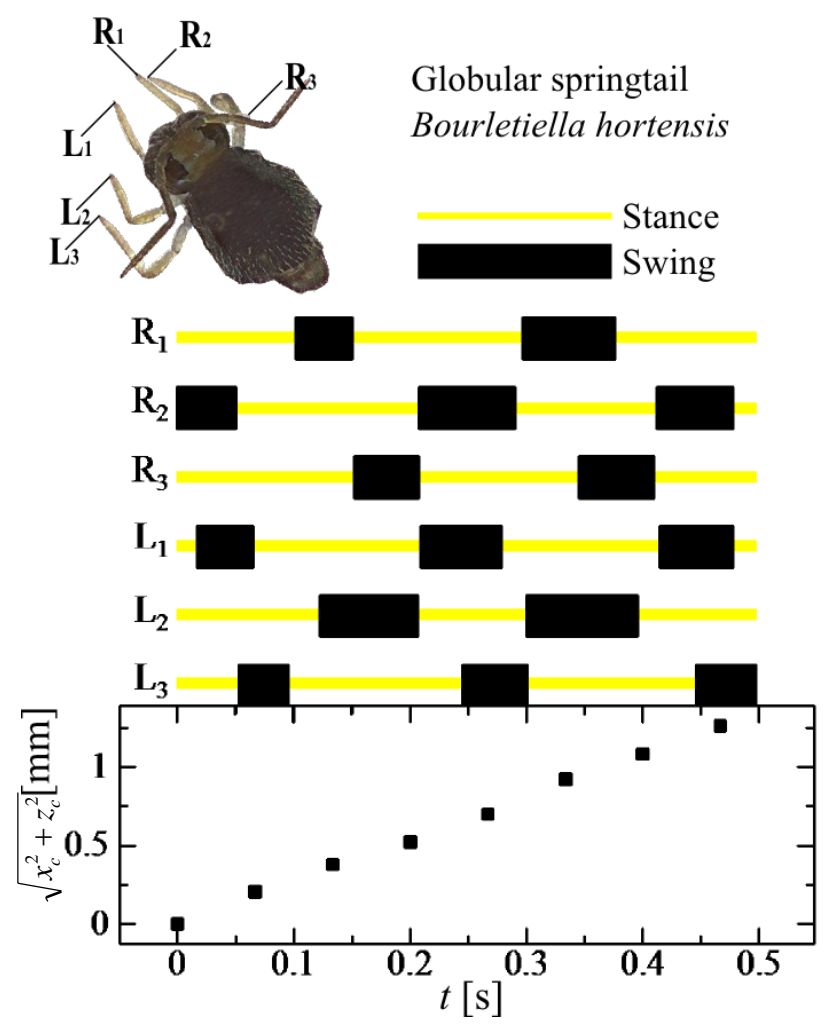

Fig. 4. Gait pattern in vertical climbing.

and $z_{c}$ is the vertical distance in the climbing of the springtail. In this experiment, the locomotive velocity in the springtail climbing $V_{c}$ was obtained as follows:

$$
V_{c}=\frac{\sqrt{x_{c}^{2}+z_{c}^{2}}}{t} \approx 2.7 \times 10^{-3} \mathrm{~m} / \mathrm{s}
$$

This value is quite distinct from the jumping velocity $V_{j}$, that is, $V_{j}>>V_{c}$.

In general, many insects are capable of climbing and walking upside down on diverse substrates using adhesive structures on their legs [11]. Dynamics of rapid vertical climbing in cockroaches was reported [12]. Unfortunately, adhesive mechanism during climbing of the springtail is not known.

\section{KINEMATIC DESCRIPTION OF SPRINGTAIL JUMP}

Globular springtails are able to jump by a special leaping organ (or furcula) as a means of escaping from predators. Springtails jump by rapidly extending their furcula that is normally folded forward under the abdomen [13]. Jumping is a common means of escaping rapidly from predators or increasing the speed of locomotion [14]. In general, animals use diverse strategies to reduce the energetically expensive cost of locomotion [1]. The energy costs of swimming, flying, and running related to the animal's size or weight, that is, the cost of locomotion increases with the decrease of body weight of animals [15].
In this chapter, jumping kinematics such as the takeoff velocity of springtail and the forces acting on the body were considered. In general, when a threedimensional body is immersed in a fluid and is in relative motion with respect to it, the forces acting on the body are described by the following equations:

$$
\begin{aligned}
& F_{L}=\frac{1}{2} \rho_{a} V^{2} C_{L} A \\
& F_{D}=\frac{1}{2} \rho_{a} V^{2} C_{D} A \\
& F_{S}=\frac{1}{2} \rho_{a} V^{2} C_{S} A
\end{aligned}
$$

where $F_{L}$ is the lift force, $F_{D}$ is the drag force, $F_{S}$ is the side force, $\rho_{a}$ is the density of fluid (air), $V$ is the velocity, $A$ is the frontal area, and $C_{L}, C_{D}$, and $C_{S}$ are the coefficients of lift, drag, and side force respectively.

If the elastically-stored energy in the springtail's furcula $E$ is completely converted into kinetic energy, the take-off velocity $V$ of the springtail is described as follows:

$$
V=\left(\frac{2 E}{m_{s}}\right)^{\frac{1}{2}}
$$

where $m_{s}$ is the mass of the body. The height in a vertical jump $H_{V}$ is given by Eq.(5):

$$
H_{V}=\frac{E}{m_{s} g}=\frac{V^{2}}{2 g}
$$

where $g$ is the gravitational acceleration. The time rate of change of the linear moment of the insect is equal to the resultant force acting on the center of mass. If the upward force generated by the furcula acts on the center of mass for the time interval $t$, the velocity $V$ and the mean acceleration $\alpha$ is given as follows [16]:

$$
\begin{aligned}
& V=\frac{F_{j} t}{m_{s}} \\
& \alpha=\frac{V}{t}=\frac{F_{j}}{m_{s}}=\frac{V^{2}}{2 \xi}
\end{aligned}
$$

where $F_{j}$ is the jumping force, $t$ is the time, and $\xi$ is the distance over which the acceleration occurs.

Assuming that a take-off angle is $\theta$ against the horizontal ground, then the maximum height $H_{m}$ and distance achieved $S_{h}$ are given as follows:

$$
H_{m}=\frac{V^{2} \sin ^{2} \theta}{2 g}
$$




$$
S_{h}=\frac{2 V^{2} \cos \theta \sin \theta}{g}=\frac{V^{2} \sin 2 \theta}{g}
$$

In the analytical model we approximate the springtail by a sphere with the same volume as the prolate spheroid. In the same manner as the jump analysis in planktonic copepoda [17], the radius of the sphere $R$ is given as follows:

$$
R=\lambda^{\frac{2}{3}}\left(\frac{L}{2}\right)
$$

where $\lambda$ is the aspect ratio of springtail body, and $L$ is the body length. The equation of motion of the vertically projected body for the upward-moving phase may be written as follows:

$$
\left(m_{s}+m_{\text {add }}\right) \frac{d V}{d t}+F_{D}+m_{s} g=0
$$

where $m_{\text {add }}$ is the mass of the displaced surrounding fluid (air). We describe $m_{s}$ and $m_{\text {add }}$ for the spherical body as follows:

$$
\begin{aligned}
& m_{s}=\frac{4}{3} \pi \rho_{s} R^{3} \\
& m_{\text {add }}=\frac{2}{3} \pi \rho_{a} R^{3}
\end{aligned}
$$

where $\rho_{s}$ is the density of springtail.

In Eq.(11), assuming the motion of the axisymmetric object, $F_{L}$ and $F_{S}$ were ignored. In this paper, the mass of springtail was $m_{s} \cong 1.3 \times 10^{-7} \mathrm{~kg}$, therefore $m_{\text {add }}$ was $m_{\text {add }} \cong 6.3 \times 10^{-11} \mathrm{~kg}$. Science $m_{s}>>m_{\text {add }}, m_{\text {add }}$ can be neglected. The second term on the left-hand side of Eq.(11) is the hydrodynamic drag

$$
F_{D}=\frac{1}{2} \pi C_{D} \rho_{a} R^{2} V^{2}
$$

The drag coefficient $C_{D}$ depends on the Reynolds number $R e$ :

$$
R e=\frac{2 \rho R V}{\mu}=\frac{2 R V}{v}
$$

where $\mu$ is the coefficient of viscosity and $v$ is the coefficient of kinematic viscosity. As described later, the Reynolds number ranged $R e<500$ in the springtail jump. For Allen flow $(0.2<R e<500)$, the drag coefficient $C_{D}$ may be written as follows:

$$
C_{D}=\frac{5 \pi}{4 \sqrt{R e}}
$$

The solution of Eq.(11) for the case of constant $R e$ gives the jump height $H_{s}$ as follows:

$$
H_{S}=\frac{4 \sqrt{\operatorname{Re}} m_{s}}{5 \pi^{2} \rho_{a} R^{2}} \ln \left[\frac{5 \pi^{2} \rho_{a} R^{2} V^{2}}{8 m_{s} g \sqrt{R e}}+1\right]
$$

Eq.(17) shows that the height of springtail jump depends on the Reynolds number and the mass of springtail.

\section{EXPERIMENTAL RESULTS AND DISCUSSION ON JUMPING}

\section{A. Jumping Movements}

The sequence of events during a jump of tiny springtail $(L=1.0 \mathrm{~mm})$ is shown in Fig.5 (a) by the multiple photograph method. In this case, the springtail jumped at the angle $\theta \cong 86$ degree to the ground. The arrows in Fig.5 (a) show the direction of springtail

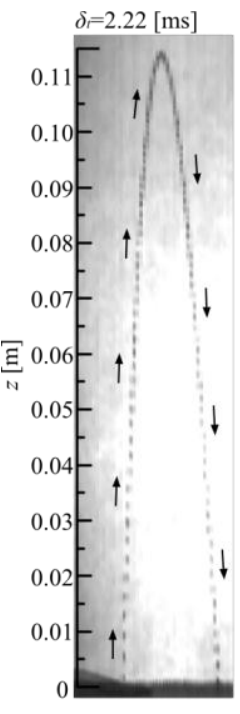

(a) Whole trajectory

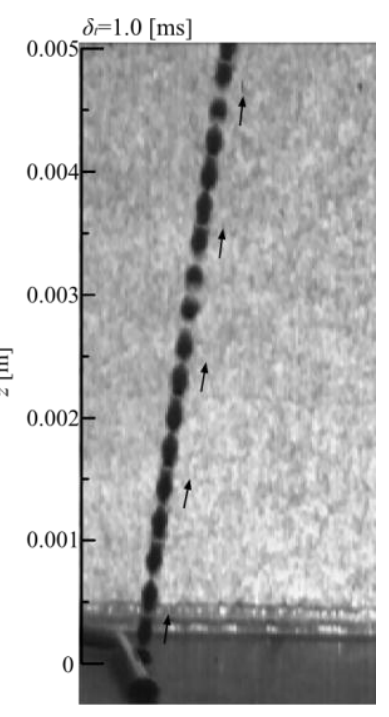

(b) Initial part
Fig. 5. Jump trajectories of two springtails.

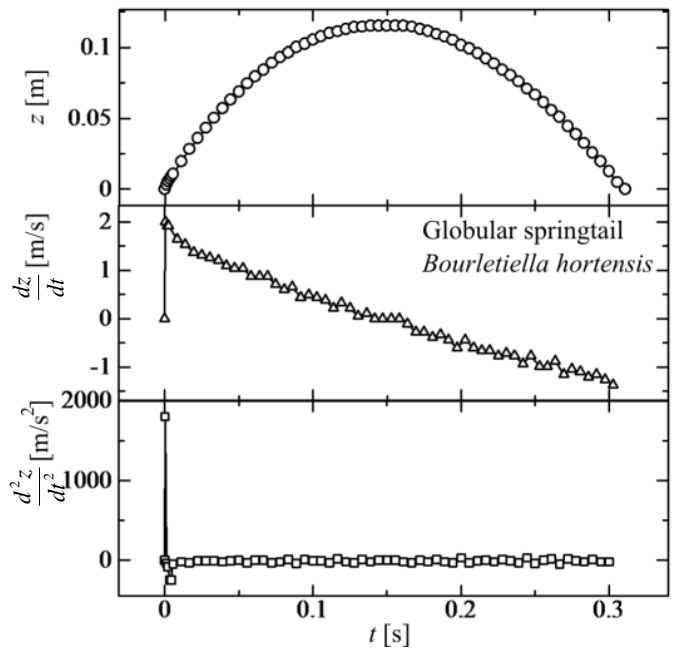

Fig. 6. Position, velocity, and acceleration as function of time during a jump. 


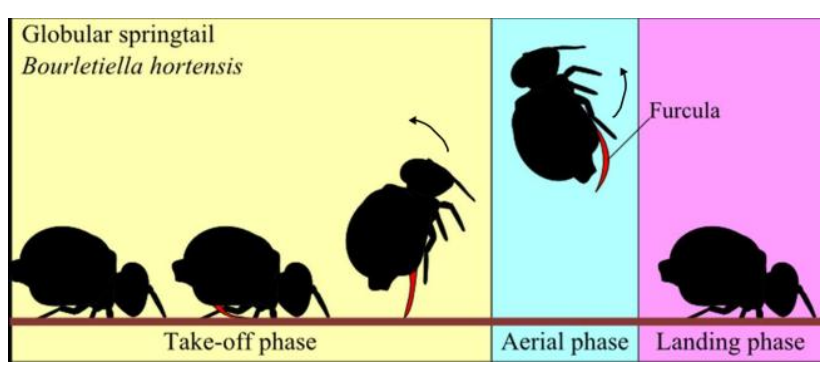

Fig. 7. Three phases in the springtail jump.

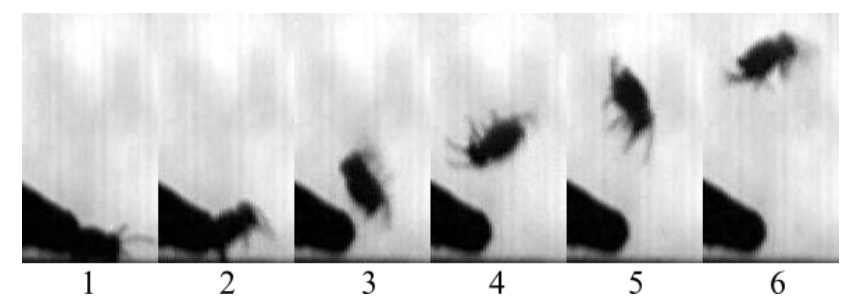

Fig. 8. Body rotation of springtail during the jump.

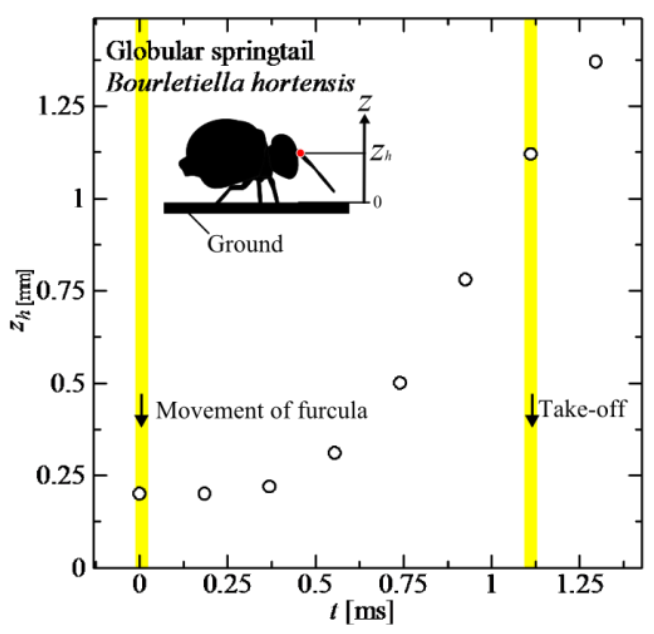

Fig. 9. Distance moved by springtail head in the take-off.

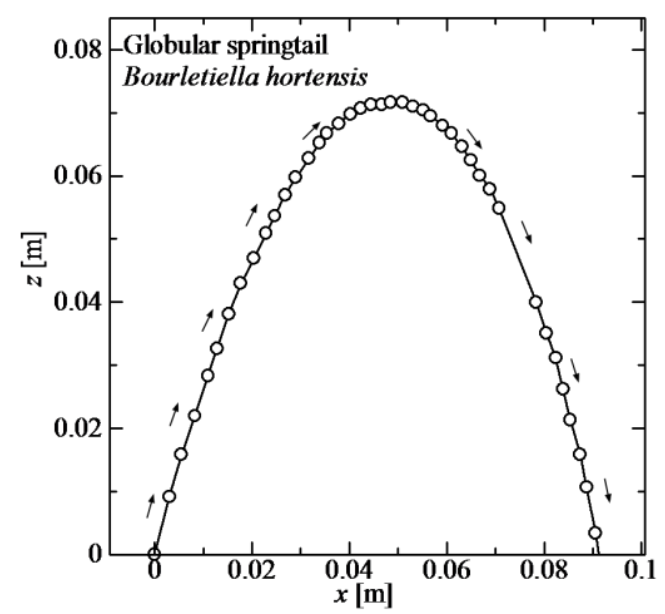

Fig. 10. Jump trajectory with larger horizontal distance.

movement, and the symbol $\delta_{t}$ shows the time interval of data plots for jumping motion. Figure 5 (b) shows the initial stage of jumping with the body rotation of springtail. The video movie of Fig.5 (b) is attached to the present paper (Movie S1). The change in position, velocity, and acceleration as function of time is shown in Fig.6. It can be seen from Fig.6 that the maximum height is $H_{m} \cong 0.115 \mathrm{~m}$, the take-off velocity is $V \approx 2.0 \mathrm{~m} / \mathrm{s}$, and the acceleration is $d^{2} z / d t^{2} \approx 1.8 \times 10^{3}$ $\mathrm{m} / \mathrm{s}^{2}$. In this experiment, the mass of springtail was $m_{s}=01.3 \times 10^{-6} \mathrm{~kg}$. The height in the vertical jump can be calculated from Eq.(5), that is,

$$
H_{V}=\frac{V^{2}}{2 g}=\frac{(2.0)^{2}}{2 \times 9.8}=0.20 \mathrm{~m}
$$

The comparison between the maximum height $H_{m}$ in Fig. 6 and the vertical jump height $H_{V}$ gives $H_{V}-H_{m}=0.08$ m. This difference $\left(H_{V}-H_{m}\right)$ is mainly brought by the effect of drag $F_{D}$ acting on the springtail. The jumping force $F_{j}$ can be calculated from Eq.(7) as follows:

$$
F_{j}=m_{s} \frac{d^{2} z}{d t^{2}}=0.13 \times 10^{-6} \times 1.8 \times 10^{3}=0.23 \times 10^{-3} \mathrm{~N}
$$

On the basis of the actions and positions of the body and furcula, three phases in the jump were identified from the frames of high-speed video movies (Fig.7): (i) the take-off phase, which last from the start of furcula movement to the point at which the springtail leaves the ground, (ii) the aerial phase, which lasts from this point to the first touching point of the ground, (iii) the landing phase (or the bouncing phase), measured as the time from the first contact with the ground. At the take-off phase, the tail springs strikes the ground. The body of the springtail is accelerated through this strike motion. Figure 8 displays a sequence of images for the early stage of springtail jump. The time interval between successive images in Fig. 8 is $\delta_{t}=0.48 \mathrm{~ms}$. The selected frames from the movie prove the body rotation of springtail during the jump. The direction in body rotation is the backward somersault.

Figure 9 shows the details of the take-off phase which was recorded with $27000 \mathrm{fps}$. In Fig.9, the head point $z_{h}$ was defined as the root of an antenna of the sprigtail. The change of position $z_{h}$ corresponds to the movement during the take-off of springtail. The contact time of the furcula is about $t=1.12 \mathrm{~ms}$. Using the contact time, Eq.(19), and Eq.(6), we can calculate the jump velocity as follows:

$$
V=\frac{F_{j} t}{m_{s}}=\frac{0.23 \times 10^{-3} \times 1.12 \times 10^{-3}}{0.13 \times 10^{-6}}=1.98 \mathrm{~m} / \mathrm{s}
$$

The value in Eq.(20) agrees the experimental value in Fig.6. In Fig.5 (a), the time required to reach the maximum height $t_{a}$ is $t_{a}=0.150 \mathrm{~s}$. On the other hand, the time required to reach the ground from the maximum height $t_{d}$ is $t_{d}=0.161 \mathrm{~s}$. This fact suggests the existence of locomotion difference between ascending and descending in the aerial phase of springtail jump. 

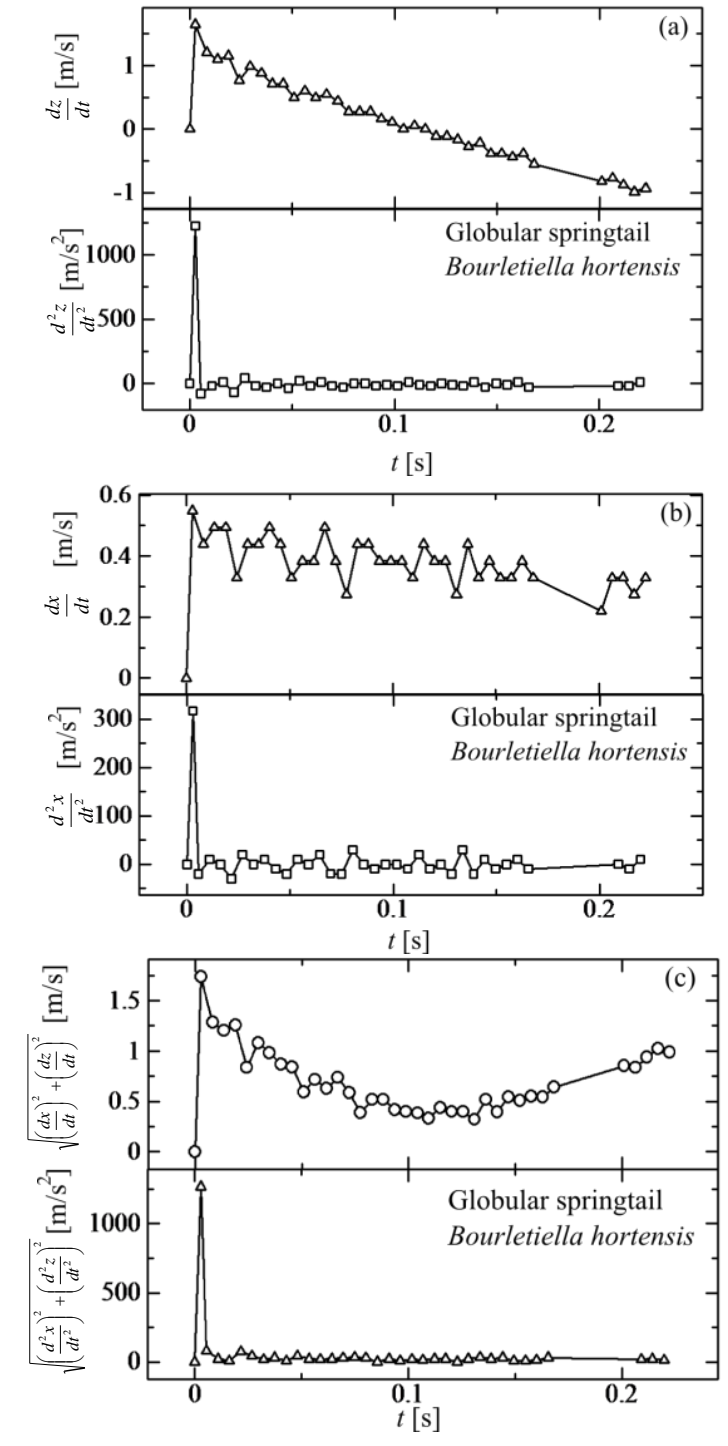

Fig. 11. Velocity and acceleration as function of time during a jump.

The drag force $F_{D}$ in Eq.(2) is caused by the flow of air around the springtail. The drag force is a function of the air velocity and density, frontal area and drag coefficient. The springtail may be adjusting the drag coefficient according to ascending and descending in the aerial phase. Figure 5 (b) and Fig.8 show body rotation of the springtail in aerial phase. In Fig.3 (b), the rotation of springtail shows 417 times per second. A computer model with the body rotation (spin rate: -8.5 37 revolutions/ second) has been used to analyze jump kinematics and energetics [13]. However, the springtail showed higher revolutions in this experiment. Figure 8 shows the initial stage in springtail jump. It can be seen from Fig. 8 that the springtail of $1 \mathrm{~mm}$ body length spins through the air. In this state, the springtail spins backward rapidly through the air. The rotation of the springtail produces an air circulation around the body. This fact suggests that globular springtails use the Magnus effect to create lift in Eq.(1). However, the springtail must be wasted energy by spinning. It can be considered that the spring tails keep their jump under control in the height and direction with spinning.

\section{B. Jumping with larger horizontal distance}

Figure 10 shows the example of the springtail jump with larger horizontal distance (in the case of $H_{m}<S_{h}$ ). In Fig.10, the vertical maximum height is $H_{m}=0.072 \mathrm{~m}$, and the horizontal distance is $S_{h}=0.091 \mathrm{~m}$. Figure 11 shows the velocity components, acceleration components, and those magnitudes in the jump case of Fig.10. In Fig.11, the maximum vertical velocity is $(d z / d t)_{\max }=1.65 \mathrm{~m} / \mathrm{s}$, and the maximum horizontal velocity is $(d x / d t)_{\max }=0.55 \mathrm{~m} / \mathrm{s}$. In this case, the take-off velocity is $V=1.74 \mathrm{~m} / \mathrm{s}$. Comparing Fig.6 with Fig.11, it can be seen that the horizontal jump leads the decrease in the take-off velocity and acceleration.

\section{CONCLUSIONS}

The jumping analysis of the globular springtail was conducted using the high-speed video camera system. The gait pattern of the springtail in vertical climbing was also examined. The results obtained are summarized as follows:

(1) The biological kinematic data of a jumping globular springtail were obtained. The maximum acceleration of the center of gravity reached $1.8 \times 10^{3}$ $\mathrm{m} / \mathrm{s}^{2}$, causing the jump velocity of about $2.0 \mathrm{~m} / \mathrm{s}$.

(2) The locomotive velocity in the springtail climbing was obtained as $V_{c}=2.7 \times 10^{-3} \mathrm{~m} / \mathrm{s}$. This climbing velocity is very lower than the jumping velocity.

(3) The globular springtail showed the jump with the high-speed body rotation. The number of revolutions reached 417 times/second.

\section{ACKNOWLEDGMENT}

This work was partly supported by JSPS KAKENHI Grant Number 22560173.

\section{References}

[1] Glass, A. C., Jorgensen, S. J., Liebsch, N., Sala, J.E., Norman, B., Hays, G. C., Quintana, F., Grundy, E., Campagna, C., Trites, A.W., Block, B. A. and Wilson, R. P., Convergent Evolution in Locomotory Patterns of Flying and Swimming Animals, Nature Communications, DOI: 10.1038(2011), pp.1-7.

[2] Bennet-Clark, H. C, and Lucey, E. C. A., The Jump of the Flea: A Study of the Energetics and a Model of the Mechanism, Journal of Experimental Biology, Vol.47(1967), pp.59-76.

[3] Bennet-Clark, H. C., The Energetics of the Jump of the Locust Schistocerca gregaria, Journal of Experimental Biology, Vol.63(1975), pp.53-83.

[4] Alexander. R. McN., Leg Design and Jumping Technique for Humans, Other Vertebrates and Insects, Philosophical Transactions of the Royal Society B, Vol.347(1995), pp.235-248.

[5] Burrows, M., Jumping Performance of Planthoppers (Hemiptera, Issidae), Journal of Experimental Biology, Vol.212(2009), pp.2844-2855.

[6] Christian, E., The Jump of the Springtails, Naturwissenschaften, Vol.65 (1978), pp.495-496. 
[7] Zettel, J., Zettel, U. and Egger, B., Jumping Technique and Climbing Behaviour of the Collembolan Ceratophysella sigillata (Collembola: Hypogastruridae), European Journal of Entomology, Vol.97(2000), pp.4145 .

[8] Hopkin, S.P., Collembola, Encyclopedia of Soil Science, (2002), pp.207-210.

[9] Full, R. J. and Tu, M.S., Mechanics of a Rapid Running Insect: Two-, Four and Six-Legged Locomotion, Journal of Experimental Biology, Vol.156(1991), pp. 215-231.

[10] Akimoto, K., Watanabe, S. and Yano, M., An Insect Robot Controlled by the Emergence of Gait Patterns, Artif Life Robotics, Vol.3(1999), pp.102-105.

[11] Clemente, C. J., Dirks, J. -H., Barbero, D. R., Steiner, U. And Federle, W., Friction Ridges in Cockroach Climbing Pads: Anisotropy of Shear Stress Measured on Transparent, Microstructured Substrates, Journal of Comparative Physiology A, Vol.195(2009), pp.805-814
[12] Goldman, D. I., Chen, T. s., Dudek, D. M. and Full, R. J., Dynamics of Rapid Vertical Climbing in Cockroaches Reveals a Template, Journal of Experimental Biology, Vol.209(2006), pp.2990-3000.

[13] Brackenbury, J. and Hunt, H., Jumping in Sprintails: Mechanism and dynamics, Journal of Zoology London, Vol.229(1993), pp.217-236.

[14] Burrows, M., Jumping Strategies and Performance in Shore Bugs (Hemiptera, Heteroptera, Saldidae), Journal of Experimental Biology, Vol.212(2009), pp.106-115.

[15] Schmidt-Nielsen, K., Locomotion: Energy Cost of Swimming, Flying, and Running, Science, Vol.177(1972), pp.222-228.

[16] Azuma, A., The Biokinetics of Flying and Swimming, (1992), pp.9-18, Springer-Verlag.

[17] Kirboe, T., Andersen, A., Langlois, V.J. and Jakobsen, H., Unsteady Motion: Escape Jumps in Planktonic Copepods, Their Kinematics and Energetics, Journal of the Royal Society Interface, Vol.7 (2010), pp. 1591-1602. 